\title{
Adapting, piloting and evaluating complex public health interventions: lessons learned from the Nurse-Family Partnership in Canadian public health settings
}

\author{
S. M. Jack, RN, PhD (1, 2, 3); N. Catherine, PhD (4); A. Gonzalez, PhD (2, 5); H. L. MacMillan, MD (2, 3, 5, 6); \\ D. Sheehan, RN, MSW (4); C. Waddell, MD (4); for the British Columbia Healthy Connections Project Scientific Team
}

This article has been peer reviewed.

Tweet this article

\section{Abstract}

Introduction: The Nurse-Family Partnership (NFP) is a home-visit program for young and first-time, socially and economically disadvantaged mothers. Evidence from three United States randomized controlled trials (RCTs) on the effectiveness of this intervention at improving pregnancy outcomes, improving child health and development, and increasing maternal economic self-sufficiency is robust. However, the effectiveness of the NFP in Canada, with its different health and social care context, needs to be determined. The purpose of this article is to describe the complex process for moving the NFP from the research arena to full implementation in Canada.

Methods: This process of evaluation in Canada includes (1) adapting the intervention; (2) piloting the intervention in small-scale feasibility and acceptability studies; and (3) conducting an RCT and process evaluation through a study called the British Columbia Healthy Connections Project (BCHCP). This large-scale evaluation also creates an opportunity to expand the NFP evidence base by conducting an additional study to examine potential biological mechanisms linking intervention and behavioural outcomes in children.

Results: Adaptation of the NFP home-visit materials is a continuous process. A pilot project determined that it was feasible to enrol eligible women into the NFP. This pilot also determined that, in Canada, it was most appropriate for public health agencies to implement the NFP and for public health nurses to deliver the intervention. Finally, the pilot showed that this intensive home-visit program was acceptable to clients, their family members and health care providers.

Through the BCHCP, the next steps-the RCT and process evaluation-are currently underway. The BCHCP will also set the foundation for long-term evaluation of key public health outcomes in a highly vulnerable population of families.

Keywords: intervention studies, prevention, public health nursing, home visits, child maltreatment

\section{Introduction}

The goal of delivering evidence-based public health interventions that focus on promoting health and preventing poor health and social outcomes is preceded by a complex process of developing, piloting and evaluating promising interventions or programs. The
Key findings

- The effectiveness of the Nurse-Family Partnership program has been established in the United States.

- This complex public health intervention, which improves the lives of vulnerable first-time mothers and their families, requires adaptation, piloting and an evaluation of both outcomes and process in this new context prior to implementation in Canada.

purpose of this article is to describe the multistep process underway in Canada to adapt, pilot and evaluate the Nurse-Family Partnership (NFP) home visitation program. This public health intervention was shown to be effective at improving pregnancy outcomes, child health and development and maternal economic self-sufficiency among socially and economically disadvantaged first-time mothers and their children in the United States (US). However, the effectiveness of this home visitation program has yet to be established within the Canadian public health and social care systems. A key priority for evaluating and implementing the NFP in Canada is to measure and address important child mental health outcomes, including the prevention of child abuse and neglect.

Child maltreatment, which includes all types of child abuse and neglect, remains a major public health problem in Canada,

Author references:

1. School of Nursing, McMaster University, Hamilton, Ontario, Canada

2. Offord Centre for Child Studies, McMaster University, Hamilton, Ontario, Canada

3. Department of Clinical Epidemiology \& Biostatistics, McMaster University, Hamilton, Ontario, Canada

4. Children's Health Policy Centre, Simon Fraser University, Vancouver, British Columbia

5. Department of Psychiatry and Behavioural Neurosciences, McMaster University, Hamilton, Ontario, Canada

6. Department of Pediatrics, McMaster University, Hamilton, Ontario, Canada

Correspondence: Susan Jack, HSC 2J32, 1280 Main Street West, School of Nursing, McMaster University, Hamilton, ON L8S 4K1; Tel: 905-525-9140, ext. 26383; Email: jacksm@mcmaster.ca 
despite efforts to reduce its occurrence. Results from a recent national survey suggest that approximately one-third of the Canadian population have experienced one or more types of child maltreatment. ${ }^{1}$ Afifi et al. ${ }^{1}$ also reported that there is a dose-response relationship between exposure to child abuse and the development of mental health conditions.

Home visiting has been promoted for more than three decades as an approach to preventing child abuse and neglect. During the 1980s, following the pilot results of the Hawaii Healthy Start program, ${ }^{2}$ home visiting by paraprofessionals was widely implemented in Canada and the US; it was considered the primary approach to preventing child maltreatment. With greater emphasis on rigorous methods for evaluating outcomes over the next two decades, it became clear that home visiting programs were not uniformly effective in preventing child maltreatment and associated outcomes such as injuries. The NFP, however, was the one with the best evidence for preventing child abuse and neglect, based on official child protection reports and associated outcomes such as injuries. ${ }^{3}$

The NFP is a program aimed at socially and economically disadvantaged first-time mothers. It begins during their pregnancy and continues until the child is two years old. The findings from three US randomized controlled trials (RCTs) have shown the NFP as having a wide range of benefits for maternal and child health, including prevention of child abuse and neglect. ${ }^{4}$ Most recently, results of a follow-up of the RCT conducted in Memphis showed a reduction in all-cause mortality among visited mothers and in preventable-cause child mortality when the children reached 20 years of age. ${ }^{5}$ In addition, in 2004 the Washington State Institute for Public Policy estimated, based on data from the three RCTs, that society experiences a return on investment of $\$ 17000$ USD for every family served by the NFP. ${ }^{6}$

\section{Methods}

Following these positive findings, efforts began more than a decade ago to bring NFP to Canada as a strategy to prevent child maltreatment and improve children's mental health and development as well as the lives of disadvantaged first-time mothers. However, it could not be assumed that the positive US findings would be replicated in Canada, especially in light of our policy, socioeconomic, demographic and geographic differences. For example, Canada offers more generous baseline public programs including health care, income supports and child benefits, with near-universal coverage for the lowest-income mothers. In keeping with these concerns, Dr. David Olds, the program developer, requires that the NFP not be implemented elsewhere without first undergoing a trial to examine its effectiveness outside of the US.

Olds et al. $^{7}$ are strongly committed to adapting the NFP to societies outside the US as long as the outcomes are rigorously evaluated to ensure that the program benefits disadvantaged mothers and children. The Prevention Research Center at the University of Colorado Denver asks that international sites implementing the NFP agree to follow a four-step protocol, ${ }^{8}$ outlined in Table 1 . This process was adhered to when the Family-Nurse Partnership Programme was implemented and evaluated in England. First, a formative evaluation documented the process of implementing the intervention in 10 pilot sites with a focus on determining site fidelity to the model elements. ${ }^{9}$ This study was followed by a large-scale RCT to determine overall program effectiveness in England, with results expected to be delivered in 2015. Likewise, in the Netherlands, where the NFP is called VoorZorg, similar steps were undertaken to translate and adapt the US guidelines to this new society, followed by a pilot study to assess treatment integrity and the feasibility of delivering the program in this context. ${ }^{10}$ This work was followed by an RCT, with published results demonstrating the effectiveness of the NFP, compared to usual care, in reducing intimate partner violence among home-visited mothers. ${ }^{11}$

Table 2 shows a timeline of all activities that relate to the process of adapting, piloting and evaluating the NFP in Canada.

\section{Results}

\section{Adaptation of the Nurse-Family Partnership for use in Canada}

A strategic first step in introducing the NFP to Canada was the development of a partnership between researchers at McMaster University, Hamilton, Ontario, and senior decision-makers at Hamilton Public Health Services, Hamilton, Ontario. This was the ideal setting to conduct and implement an NFP pilot study because of strong community support for the program, a public health unit with strong evidence-based programs and a partnership with a research team at McMaster University with expertise in conducting evaluations of child maltreatment and family violence interventions. It was determined that the NFP should be delivered through public health and by public health nurses (PHNs) because PHNs historically visit the homes of vulnerable families in the perinatal period. PHNs in most Canadian jurisdictions hold a baccalaureate degree in nursing, which meets one of the core fidelity elements of the NFP intervention model. Identification of a program champion at the local public health unit level was essential for reallocating a portion of existing provincial home-visit funding towards implementing and piloting of the program. ${ }^{12}$

Adapting the NFP materials, including visit-to-visit guidelines (recommendations to guide the content of each visit), nurse

TABLE 1

Protocol for international replication and evaluation of the Nurse-Family Partnership

1. Adapt the NFP program to local contexts and populations while ensuring fidelity to the NFP model elements.

2. Assess feasibility and acceptability of the adapted program in a small-scale pilot study.

3. Evaluate the adapted program in a large-scale RCT.

4. Expand the adapted program within that society if the evaluation shows significant positive outcomes.

Abbreviations: NFP, Nurse-Family Partnership; RCT, randomized control trial. 
TABLE 2

Timeline for adapting, piloting and evaluating the Nurse-Family Partnership in Canada

\begin{tabular}{|c|c|c|c|}
\hline Years & Evaluation component & Location & Activities \\
\hline 2008-11 & Phase 1: Adaptation & ON & NFP guidelines adapted to include Canadian standards of evidence and updated content. \\
\hline 2008-12 & Phase 2, Step 1: Feasibility study & ON & $\begin{array}{l}\text { Pilot study to test procedures for recruitment and retention and instruments for collecting } \\
\text { clinical and interview data from participants. }\end{array}$ \\
\hline $2008-12$ & Phase 2, Step 2: Acceptability study & ON & $\begin{array}{l}\text { A qualitative case study to explore the acceptability of the NFP to clients, their families, PHNs } \\
\text { and community stakeholders. }\end{array}$ \\
\hline 2011-ongoing & $\begin{array}{l}\text { Phase 3, Step 1: Preparation for } \\
\text { RCT adaptation (version } 2.0 \text { ) }\end{array}$ & $\mathrm{ON}$ and $\mathrm{BC}$ & $\begin{array}{l}\text { Using feedback from Hamilton PHNs and BC nurse leaders, update and revise the NFP } \\
\text { Canadian guidelines; further enhance with integration of intimate partner violence and DANCE } \\
\text { nurse education modules, guideline facilitators and nurse instructions. }\end{array}$ \\
\hline 2011-14 & $\begin{array}{l}\text { Phase 3, Step 2: Preparation for RCT } \\
\text { PHN/Supervisor education }\end{array}$ & $\mathrm{BC}$ & $\begin{array}{l}\text { Hiring of PHNs and supervisors; complete nurse education; delivery of the NFP to "guiding } \\
\text { clients." }\end{array}$ \\
\hline 2013-18 & Phase 3: Large-scale RCT & $\mathrm{BC}$ & $\begin{array}{l}\text { Identify eligible pregnant women to enroll in BCHCP RCT to compare the NFP to existing } \\
\text { services in reducing childhood injuries (primary outcome). }\end{array}$ \\
\hline 2013-18 & Phase 3: Process Evaluation & $\mathrm{BC}$ & $\begin{array}{l}\text { Consent a purposeful sample of senior public health managers and all NFP PHNs and } \\
\text { supervisors to document process for implementing and delivering NFP. }\end{array}$ \\
\hline 2014-18 & Phase 3: Healthy Foundations Study & $\mathrm{BC}$ & $\begin{array}{l}\text { Identify } 300 \text { mother-infant dyads to enroll to measure and determine effect of NFP on } \\
\text { biological mechanisms linking intervention and behavioural outcomes in children. }\end{array}$ \\
\hline
\end{tabular}

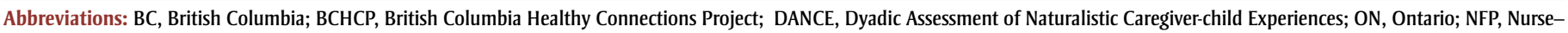
Family Partnership; PHN, public health nurses; RCT, randomized control trial.

instructions, client worksheets and other resources for use during home visits in Canada, has been an ongoing collaborative effort between public health management, the front-line PHNs who provide feedback based on their clinical experiences delivering the NFP, and nurse-researchers (SJ, DS) who integrate nurses' clinical practice knowledge with existing best practices and research evidence. To date, specific content adaptations to the US materials have included:

(1) integration of Canadian standards of practice and best practice guidelines on topics such as immunization schedules, food and nutritional intake recommendations, and injury prevention guidelines;

(2) augmentation of materials to meet identified local needs or priority issues, including meeting recommendations from the Baby-Friendly Initiative to promote breastfeeding; ${ }^{13}$

(3) integration of new NFP innovations, including an intervention to identify and respond to intimate partner violence; ${ }^{14}$ and

(4) a tool for observing parent-child interactions called the Dyadic Assessment of Naturalistic Caregiver-child Experiences (DANCE). ${ }^{15}$

In developing guidelines that would be suitable for use in multiple provincial jurisdictions, PHNs are advised to use any local or provincial guidelines that differ from the Canadian guidelines. Considerable attention was paid at the adaptation stage to meet specific formatting requirements including (1) compliance with the Accessibility for Ontarians with Disabilities Act, 2005 ; $^{16}$ (2) conversion of measurements from imperial to metric; (3) integration or substitution of graphics to reinforce Baby-Friendly Initiative principles or to represent the diversity of clients in the Canadian context; and (4) written indication on each document that adaptations were made with permission from the NFP National Service Office. Prior to their use in the field, all adapted materials were sent to the Prevention Research Center at the University of Colorado Denver for review, feedback and approval.

\section{Assessment of NFP's feasibility and acceptability in Canada}

The objectives of the feasibility study were to assess if (1) pregnant women who met the NFP eligibility criteria could be referred and enrolled in the program; (2) the program could be delivered with fidelity to the 18 NFP model elements ${ }^{17}$ by a public health agency; (3) program-level data could be collected by the implementing agency; and (4) client-outcome data could be collected by the research team. To meet these goals, Jack et al. ${ }^{18}$ carried out a mixed methods pilot study in Hamilton between 2008 and 2012 during which 424 prenatal referrals to the Hamilton Public Health Unit were assessed for NFP eligibility. Criteria included young age ( $\leq 21$ years), low income, referral before the end of the 28th week of pregnancy and first-time birth. A total of 135 pregnant women were deemed eligible for NFP (32\% of all prenatal referrals), and 108 ( $80 \%$ of those who were eligible) consented to participate in the pilot study. Additional details of the pilot study methods are reported elsewhere. ${ }^{18}$

Of the 108 participants, 5 refused further contact and 1 was lost to follow-up prior to their baseline interview. Of the remaining 102 women, 71 completed the final interview at age 2 years. Most women (87\%) were enrolled in the program before or at 25 weeks' gestation (the international benchmark is $60 \%$ referred by 16 weeks of pregnancy) and $77 \%$ of the participating women were between 16 and 19 years of age.

Results from the pilot study demonstrated that it was feasible to provide the NFP program through public health units; have PHNs deliver the intervention; receive appropriate referrals from community partners; successfully enroll eligible participants; and to home-visit this targeted population of young, low-income first-time mothers. 
Comprehensive NFP program data were also collected via NFP nursing assessment forms that elicited systematic information on each mother's health status and health behaviours, the child's health and development, and utilization of other health and community services. A process to collect clientoutcome data by the research team was also successfully implemented.

In addition to measuring the feasibility of delivering the program, Jack et al. ${ }^{18}$ conducted a qualitative case study to explore the acceptability of delivering the NFP to families in Hamilton. In intervention research, acceptability studies are an important complement to traditional pilot studies of feasibility. Determination of an intervention's acceptability in a new context provides two benefits: (1) it allows for key stakeholders to be engaged early in the process, which may facilitate later uptake of the intervention; and (2) it identifies intervention components that may require additional adaptation to meet the needs of the targeted audience, the health care providers or the local community. ${ }^{19}$ An acceptability study focusses on an examination of the local context with the goal of understanding if, how, and under what conditions the intervention will be delivered and received by the intended target audience. In addition, acceptability studies examine if the intervention will meet the needs of the target population and the community.

With the introduction of a new public health intervention into the existing provincial home-visit program, it was important to determine community-level acceptance of and readiness for the NFP. The NFP was quite distinct from Ontario's existing provincial home-visit program in that it is targeted to first-time mothers who are socially and economically disadvantaged, it is delivered by registered nurses with a baccalaureate degree and it provides the client with a greater overall number of home visits, which start early in pregnancy.

In Hamilton, socially and economically disadvantaged mothers who participated in the home visits identified the NFP as an acceptable, early intervention public health initiative. ${ }^{20}$ At the organizational level, this intervention created an environment where PHNs could deliver holistic nursing care at their full scope of practice; develop in-depth therapeutic relationships with clients; and have the infrastructure, resources and materials to assess and intervene with clients who experience multiple, complex health and social conditions, including mental health issues and substance misuse, as well as exposure to violence. ${ }^{20}$ In addition, community professionals responsible for referring women to the program recognized that the NFP addressed an important gap in health care service delivery for families at greatest risk. ${ }^{18}$

\section{Components of the Nurse-Family Partnership Model in Canada}

The Canadian model of the NFP was built upon findings from the Jack et al. ${ }^{18}$ case study together with the requirements of the Prevention Research Center at the University of Colorado, Denver and of the NFP National Service Office. Results from the case study conducted in Hamilton informed

(1) the development of the Canadian NFP visit-to-visit guidelines including guidelines and forms for documenting nursing practices;

(2) the recommendation to deliver NFP through public health agencies by PHNs;

(3) recommendations for hiring and staffing, including caseload levels; and

(4) the importance of a staged implementation, with nurses first carrying a small caseload of "guiding clients" to work with as they engage in and complete the NFP education requirements.

See Table 3 for a summary of the components of the NFP model in Canada.

\section{Setting the stage for large-scale NFP evaluation in Canada}

The development and implementation of a large-scale study to evaluate the effectiveness of NFP in Canada required a substantive commitment of funding, research expertise, and community engagement. Attempts to expand the evaluation in Ontario were not successful. ${ }^{12}$ In 2010 ,
British Columbia (BC) announced Healthy Minds, Healthy People: A Ten-Year Plan to Address Mental Health and Substance Use. $^{21}$ In this plan, the $\mathrm{BC}$ government prioritized an intervention that featured nurse home visits to disadvantaged firsttime mothers and their children as a central initiative. B.C.'s Guiding Framework for Public Health also identified nurse visiting the homes of the most vulnerable pregnant women as a promising approach for improving health systems capacity. $^{22}$

Through their ten-year mental health plan, ${ }^{21}$ the BC Ministry of Health $(\mathrm{MoH})$ and the Ministry of Children and Family Development (MCFD) jointly invited the Children's Health Policy Centre in the Faculty of Health Sciences at Simon Fraser University (SFU) to explore options for evaluating NFP in BC with McMaster University's NFP pilot study team. The $\mathrm{MoH}$ and the MCFD also convened a Provincial Advisory Committee made up of senior representatives from five $\mathrm{BC}$ health authorities and from MCFD regions, Aboriginal organizations and other agencies, such as the Public Health Agency of Canada. These meetings created a venue to present information about the NFP and also allowed the $\mathrm{MoH}$ and the MCFD to seek province-wide consensus on proceeding with the NFP evaluation.

With funding from the $\mathrm{MoH}$ and support from the MCFD, the BC Healthy Connections Project (BCHCP) officially launched in 2012 in close collaboration with five participating health authorities (Fraser Health, Vancouver Coastal Health, Interior Health, Northern Health and Island Health). Three primary studies now make up the BCHCP: an RCT to evaluate the impact of the NFP on health and social outcomes, including early child mental health and development outcomes and maternal life course; a process evaluation to describe how the NFP is implemented and delivered; and the Healthy Foundations Study to investigate the impact of the NFP on infant biological systems and functioning. Recruitment for these studies commenced in October 2013 and is expected to continue into 2016. Data collection will continue until the participating children reach two years of age. 
TABLE 3

Nurse-Family Partnership Canada model components

\begin{tabular}{|c|c|}
\hline Components & Nurse-Family Partnership model in Canada \\
\hline Program goals & $\begin{array}{l}\text { Improve pregnancy outcomes } \\
\text { Improve child health and development } \\
\text { Improve parents' economic self-sufficiency }\end{array}$ \\
\hline Eligibility criteria & 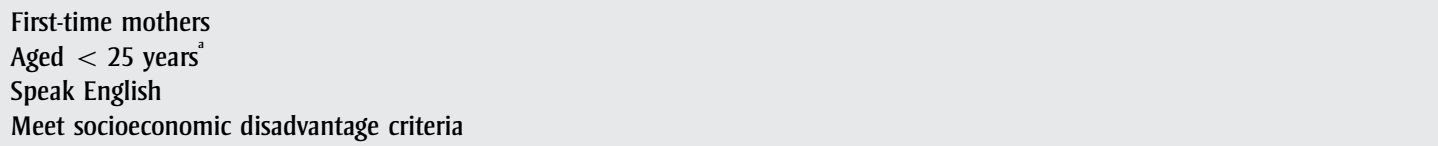 \\
\hline Referral process & Ideally referred by 16 weeks gestation; must receive first home visit before the $29^{\text {th }}$ week gestation \\
\hline $\begin{array}{l}\text { Professionals delivering home } \\
\text { visits }\end{array}$ & Public health nurses (PHNs) \\
\hline Frequency of home visits & $\begin{array}{l}\text { Prenatal: Weekly for } 4 \text { visits then bi-weekly ( } \sim 14 \text { visits) } \\
\text { Infancy: Weekly for } 6 \text { visits then bi-weekly ( } 28 \text { visits) } \\
\text { Toddler: Once every } 2 \text { weeks until } 21 \text { months and then monthly for } 3 \text { visits ( } 22 \text { visits) } \\
\text { Note: If needed, the schedule is adapted to meet the needs of each enrolled participant }\end{array}$ \\
\hline Theories used & Human ecology, attachment, self-efficacy \\
\hline Structure of visits & $\begin{array}{l}\text { Emphasis on developing a therapeutic relationship using } \\
\text { - } 5 \text { client-centred principles (client is the expert on her life; follow the client's heart's desire; only a small change is necessary; focus } \\
\text { on strengths; focus on solutions) } \\
\text { - } 6 \text { content domains (Personal Health, Life Course Development, Maternal Role, Family and Friends, Environmental Health, and } \\
\text { Health \& Human Services) } \\
\text { - motivational interviewing } \\
\text { - goal setting } \\
\text { - visit-to-visit guidelines (recommendations to guide content of each visit) } \\
\text { - client resources left in home (facilitators) } \\
\text { - data collection }\end{array}$ \\
\hline $\begin{array}{l}\text { Use of screening and } \\
\text { assessment tools }\end{array}$ & $\begin{array}{l}\text { Standard schedule of assessments. Tools used include } \\
\text { - Dyadic Assessment of Naturalistic Caregiver-child Experiences (DANCE) } \\
\text { - Ages and Stages Questionnaire } \\
\text { - Partners in Parenting Education (PIPE) } \\
\text { - Edinburgh Postpartum Depression Scale, Intimate Partner Violence } \\
\text { - NFP-specific tools }\end{array}$ \\
\hline Education & $\begin{array}{l}\text { Comprehensive core NFP education provided with a combination of self-study, team-based learning, webinars, and in-person } \\
\text { About } 20 \text { days for PHNs plus an additional } 5.5 \text { days for supervisors }\end{array}$ \\
\hline Caseload & Maximum of 20 clients for a full-time PHN \\
\hline Clinical supervision & $\begin{array}{l}\text { Structured approach to clinical and reflective supervision including weekly individual case consultations and regular home-visit } \\
\text { observations. Ratio of NFP PHN supervisors to PHNs is a maximum of 1:8 }\end{array}$ \\
\hline
\end{tabular}

Abbreviations: BCHCP, British Columbia Healthy Connections Project; NFP, Nurse-Family Partnership; PHN, public health nurse.

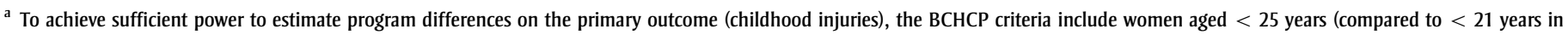
Hamilton) who are experiencing indicators of socioeconomic disadvantage associated with increased risk for child injuries.

\section{$B C H C P$ randomized controlled trial:} evaluation of NFP's effectiveness

The BCHCP includes an RCT to evaluate the effectiveness of NFP across five domains: pregnancy, birth, early child health and development, and maternal life course. Within these domains, the impact of NFP on specific outcomes for both children and mothers, as well as the associated risk and protective factors, will be evaluated. The NFP will be compared to the health and social services existing in BC ("existing services") from early pregnancy until children reach two years of age. As outlined in a recent report discussing the use of RCTs in developing public policy, conducting a trial to determine the effectiveness of the NFP in BC will ensure that the NFP group and the group receiving existing services will be as closely matched as possible. ${ }^{23}$ Evaluating the NFP with an RCT design allows for greater control over external factors that may otherwise affect findings. The BCHCP RCT study methods are briefly described here.

Women are eligible to participate in the BCHCP RCT if they are socioeconomically disadvantaged first-time mothers (i.e. low education, low income, lone parent) less than 25 years old. The goal is to enroll 1000 women and randomly assign them to either the NFP or existing services. Outcome data will be collected (through interviews and via linkages to administrative health data) at baseline and then at regular intervals until the child's second birthday. Women randomized into the "intervention" arm will be part of the NFP and will be eligible to receive existing services within their health authority; women in the "comparison" arm will receive existing services. Existing services vary across the province but may include standard primary health care services; public health programs including prenatal 
classes, pregnancy outreach and home visits by non-NFP nurses; and a variety of targeted and universal parenting and early child development programs.

The BCHCP will also be able to provide extensive data to assess BC's existing services. Service use will be tracked across a broad array of health and social sectors over the first two years of the child's life (e.g. maternal and child primary health care, standard perinatal nursing programs, parenting programs, early child development programs, child emergency room visits, child hospitalizations, child protection investigations, child foster care placements, maternal criminal justice system involvement, and family income supports). These service-use data will also be collected during BCHCP interviews and through linkages to administrative health data. Comprehensive NFP program data will be collected via NFP nursing assessment forms. Future papers will provide a detailed analysis of the use of existing services, compared with NFP, in BC.

Based on the US RCT findings, we hypothesize that the primary outcome of the NFP will be to reduce injuries from birth to age two. Secondary outcomes will include

- a reduction in prenatal substance use (i.e. tobacco and alcohol);

- an improvement in child language and cognitive development at age two years;

- an improvement in child behaviour at age two years; and

- an improvement in maternal life course at two years postpartum (i.e. number of subsequent pregnancies).

In addition, we will evaluate associated risk and protective factors (e.g. child physical health, maternal mental health, parenting, socioeconomic status, exposure to intimate partner violence). We are also setting the stage for evaluating the NFP's long-term impact across childhood and into adolescence. Service-use data will specifically enable economic evaluation. As part of defining service utilization comprehensively from a broader societal perspective, we will also gather information about services that were not accessed and the reasons for this. There is ongoing engagement with policy makers at each step of the evaluation. The common goal is to improve the lives of children in $\mathrm{BC}$, starting very early in the lifecourse.

\section{BCHCP process evaluation}

The NFP is being delivered across five of BC's health authorities, each of which is unique in its geography, population characteristics, and pre-existing culture of the partnerships and collaborations required to support the NFP at the community level. This level of complexity creates challenges for evaluating both the effectiveness of interventions and for understanding how causal mechanisms of interventions may influence intended outcomes. ${ }^{24}$ In addition to measuring trial outcomes, process evaluations are increasingly used with RCTs to contribute to the comprehensive evaluation of complex interventions. This process evaluation will also help us identify barriers to and facilitators of the successful implementation of the NFP in a range of diverse communities. Investigators will also explore strategies for resolving identified challenges related to implementation and delivery.

The primary objectives of the $\mathrm{BCHCP}$ process evaluation are to describe, and compare across BC's five participating health authorities

- how the NFP is implemented;

- if the intervention is delivered with fidelity to the NFP model elements;

- the dose of the intervention delivered and received;

- client enrolment and client and PHN retention issues;

- contextual factors at the client, health care provider, organizational and community levels that influence implementation and delivery (including a comparison between urban and rural contexts); and

- how PHNs identify and address issues related to child welfare involvement, intimate partner violence and mental health including substance misuse.

Investigators will evaluate the process evaluation using a convergent parallel mixed methods research design. ${ }^{25}$

All BC PHNs and supervisors employed by their health authority to deliver the NFP as part of their public health responsibilities will be invited to participate in the study. In each health authority, a purposeful sample of 10 to 15 public health managers/directors responsible for $\mathrm{BCHCP}$ or the NFP will also be included. To document the implementation processes within each health authority, data will be collected regularly between 2013 and 2018. All PHNs, supervisors and the NFP provincial coordinator will complete an in-depth interview (or participate in a focus group) every six months; public health managers will complete one in-depth interview annually. Documents, including individual supervisor-NFP PHN reflective supervision meetings and team meeting/ case conference summary forms, will be completed monthly by supervisors, and the data will be aggregated every 6 months. NFP program fidelity data from the $\mathrm{MoH}$ will be collected on a quarterly basis. The collection of both quantitative and qualitative data will bring together the strengths of both research traditions to compare, corroborate and explain results and variances across the five health authorities.

\section{Healthy Foundations Study}

Beyond assessing the NFP's effectiveness and the process of its implementation, we also wanted to examine potential biological mechanisms linking intervention and behavioural outcomes in the children. That adverse early experiences (i.e. childhood maltreatment and poverty) have enduring effects on cognitive, mental and physical health outcomes is well established. $^{26,27}$ Parallel lines of animal and human research show that early adversity produces long-lasting disruptions in physiology, including alterations in the stress system (hypothalamic-pituitary-adrenal axis) $)^{28,29}$ and changes in immune system function. 30,31 These changes, in turn, adversely affect brain development and health. Emerging evidence suggests that these experiences may be "biologically embedded" into molecular and genomic systems that determine expressions of vulnerability later in life. ${ }^{27}$ These experiences set developmental trajectories that are difficult to reverse later on. ${ }^{32}$ To our knowledge, no study has yet examined the impact of preventive interventions longitudinally on biological 
outcomes in mothers and their children, nor have any studies assessed whether these interventions can potentially reverse the biological embedding of disease.

In July 2013, we received funding to embed a sub-study $(\mathrm{N}=300$ dyads; $\mathrm{n}=150$ in the intervention group; $\mathrm{n}=150$ in the comparison group) within the BCHCP. This Healthy Foundations Study has three primary aims: (1) to determine whether the NFP has an effect on infant physiological function, as reflected in alterations in hypothalamic-pituitary-adrenal axis function and epigenetic markers; (2) to investigate whether the NFP has an impact on maternal prenatal cortisol levels and whether maternal prenatal cortisol levels are associated with alterations in infants' stress physiology, immune function and DNA methylation; and (3) to examine whether alterations in biological markers explain the association between the impact of the NFP on infant developmental outcomes at age two years. We will collect biological samples from both groups (NFP and existing services) and from mothers (hair) and infants (saliva) to measure levels of stress and immune function. Buccal samples are also collected from infants to examine markers that affect gene expression. A greater understanding of the biology of early adversity and the potential to reverse its detrimental effects (via NFP) provides a powerful framework with which to inform basic and applied research, practice and policy.

\section{Discussion}

The NFP holds exceptional promise as a program that can influence very early, crucial determinants of health though prenatal behaviour, parenting and child maltreatment, which in turn influences mental health and learning outcomes in early childhood and beyond. While many public health agencies are primed to implement this public health intervention, the NFP has never been tested in Canada to determine whether we can replicateand expand on-the outcomes from the US trials.

A systematic approach to adapting the US NFP materials and piloting this public health intervention for feasibility and acceptability laid the groundwork for the subsequent evaluation of effectiveness. The BCHCP is now laying the foundation to evaluate and adapt the NFP for sustained use in BC, work that could potentially be applied to successfully implement the NFP in jurisdictions across Canada. Findings from the process evaluation will inform further adaptations of the Canadian NFP guidelines and core nurse education.

The BCHCP has also created an opportunity to expand the NFP evidence base through the inclusion of the data from the Healthy Foundations Study. Findings from the process evaluation will also influence our understanding of how to adapt the service delivery model to meet the needs of families in rural and remote communities. Perhaps the most unique aspect of the BCHCP is that it is already embedded in BC's health system-as a close collaboration with child health policy makers in the MoH, MCFD and five health authorities-enabling us to evaluate the NFP's effectiveness in "real-world" conditions and also positively influence the care that mothers and children are receiving. Notably, BCHCP policy partners have already committed to adopting the NFP as an ongoing maternal-child health program in BC, should the RCT prove successful.

\section{Conclusion}

In 2004, the Prevention Research Center at the University of Colorado Denver, led by Olds, began responding to inquiries from researchers and policy makers outside the US who were interested in implementing NFP in their countries. The Prevention Research Center developed the four-step process (see Table 1) that involves helping international partners adapt and test the NFP in their own societies while maintaining fidelity to the core model elements of the program. Based on evolving understanding of prevention research, we recommend that the Prevention Research Center add the requirement that international sites incorporate a process evaluation in conjunction with the required RCT.

At present, each RCT outside the US is conducted independently and there is no formal mechanism for international NFP researchers to collaborate and consult with each other. As evaluation work on this public health intervention proceeds in different contexts, it is imperative to establish a process for collaborating internationally on different components of the program. Processes have now been put in place for countries implementing the NFP to come together to address shared issues through a Clinical Advisory Committee and a Clinical Leaders Network. Recently, a small international team was developed to support high quality implementation of the NFP in new and existing societies/ countries. In October 2014, the first meeting of representatives (education and implementation leads) from seven international NFP societies (Australia, Canada, England, Netherlands, Northern Ireland, Scotland, United States) was held in Denver, Colorado, US. The focus of the meeting included discussion and development of NFP education principles and theories, nurse competencies, and essential (or optional) education content and processes that will inform and ultimately result in an NFP Education Framework. A pre-meeting session explored the process of developing and implementing program innovations such as the DANCE and an intervention aimed at reducing intimate partner violence, both of which are part of the core NFP intervention being evaluated in BC. As this international NFP implementation work proceeds, it is hoped and anticipated that international NFP researchers can also come together to develop a collaborative network.

In $\mathrm{BC}$, we are also setting the stage for following our sample of 1000 children over 10 years or more into middle childhood and adolescence. This will allow us to fully assess the potential impact of NFP on several critical child health and public health outcomes: preventing the most common mental health problems including anxiety, depression, conduct disorder and substance misuse; improving academic achievement; and reducing maltreatment and its consequences. In addition, longterm follow-up will enable us to conduct a detailed economic evaluation of the NFP in Canada for the first time. Perhaps most importantly, we hope to build on the BCHCP to sustain and expand the childcentred approach to public health that has been initiated in $\mathrm{BC}$, leading the way for 
other provinces, and allowing us to collaborate with international partners who share our child health goals.

\section{Acknowledgements}

Members of the BCHCP Scientific Team include Ronald G. Barr, MA MDCM FRCPC (University of British Columbia); Michael Boyle, PhD (McMaster); Amiram Gafni, DSc (McMaster); Kaitlyn Hougham, MSc (Simon Fraser University); Lenora Marcellus, $\mathrm{RN}$ PhD (University of Victoria); Lawrence McCandless, PhD (Simon Fraser University); Cody Shepherd, BA (Hon) (Simon Fraser University); Lil Tonmyr, PhD (Public Health Agency of Canada); Natasha Van Borek, MScPPH (McMaster); and Colleen Varcoe, RN PhD (University of British Columbia). The Public Health Agency of Canada provided funds to adapt the NFP materials. The City of Hamilton Public Health Services funded the feasibility and acceptability studies, with additional funding from two child protection agencies (Hamilton Catholic Children's Aid Society and Hamilton Children's Aid Society), the Hamilton Community Foundation, McMaster Children's Hospital, the Ontario Ministry of Health and Long-Term Care Nursing Secretariat and the Ontario Centre for Excellence for Child and Youth Mental Health. The BC Ministry of Health funds the $\mathrm{BCHCP}$, with support from the BC Ministry of Children and Family Development and from Fraser Health Authority, Interior Health, Island Health, Northern Health and Vancouver Coastal Health. Additional funding has been provided through the Djavad Mowafaghian Foundation. The BCHCP process evaluation received additional funding from the Injury and Child Maltreatment Section, Centre for Chronic Disease Prevention, Public Health Agency of Canada. The Healthy Foundations Study is funded through the Canadian Institutes for Health Research. Harriet MacMillan is supported by the Chedoke Health Chair in Psychiatry. Charlotte Waddell is supported by a Canada Research Chair in Children's Health Policy.

\section{References}

1. Afifi TO, MacMillan HL, Boyle M, Taillieu T, Cheung K, Sareen J. Child abuse and mental disorders in Canada. CMAJ. 2014;186(9): E324-32.
2. Duggan AK, McFarlane EC, Windham AM, et al. Evaluation of Hawaii's Healthy Start program. Future Child. 1999;9(1):66-90.

3. MacMillan HL, Wathen $\mathrm{CN}$, Barlow $\mathrm{J}$, Fergusson DM, Leventhal JM, Taussig HN. Interventions to prevent child maltreatment and associated impairment. Lancet. 2009; 373:250-66.

4. Olds DL, Sadler S, Kitzman H. Programs for parents of infants and toddlers: recent evidence from randomized trials. J Child Psych Psychiatry. 2007;48:355-91.

5. Olds DL, Kitzman H, Knudtson MD, Anson E, Smith JA, Cole R. Effect of home visiting by nurses on maternal and child mortality: results of a 2-decade follow-up of a randomized clinical trial. JAMA Pediatr. 2014;168(9):800-6.

6. Aos S, Lieb R, Mayfield J, Miller M, Pennucci A. Benefits and costs of prevention and early intervention programs for youth. Olympia (WA): Washington State Institute for Public Policy; 2004.

7. Olds DL, Hill PL, O’Brien R, Racine D, Moritz P. Taking preventive intervention to scale: The Nurse-Family Partnership. Cogn Behav Pract. 2003;10:278-90

8. Prevention Research Center for Family and Child Health. Nurse-Family Partnership international program [Internet]. Denver (CO): University of Colorado Denver; [cited 2014 Dec 4]. Available from: http://www. ucdenver.edu/academics/colleges/medicalschool/departments/pediatrics/research/ programs/prc/research/international/Pages/ international.aspx.

9. Barnes J. From evidence-base to practice: implementation of the Nurse Family Partnership programme in England. J Child Serv. 2010;5:4-17.

10. Mejdoubi J, van den Heijkant S, Struijf E, van Leerdam F, HiraSing R, Crijnen A. Addressing risk factors for child abuse among high risk pregnant women: design of a randomised controlled trial of the nurse family partnership in Dutch preventive health care. BMC Public Health. 2011;11:823.

11. Mejdoubi J, van den Heijkant S, van Leerdam FJ, Heymans MW, Hirasing RA, Crijnen AA. Effect of nurse home visits vs usual care on reducing intimate partner violence in young high-risk pregnant women: a randomized controlled trial. PLoS ONE. 2013;8(10):e78185.
12. Jack SM, MacMillan HL. Adaptation and evaluation of the Nurse-Family Partnership in Canada [Internet]. Early Childhood Matters. 2014 [cited 2014 Nov 24]; 43-46. Available from: http://earlychildhoodmagazine.org/ adaptation-and-evaluation-of-the-nursefamily-partnership-in-canada/.

13. Pound CM, Unger SL. The Baby-Friendly Initiative: protecting, promoting and supporting breastfeeding. Paediatr Child Health. 2012;17(6):317-21.

14. Jack SM, Ford-Gilboe M, Wathen CN, et al. Development of a nurse home visitation intervention for intimate partner violence. BMC Health Serv Res. 2012;12:50.

15. Olds D, Donelan-McCall N, O’Brien R, et al. Improving the Nurse-Family Partnership in community practice. Pediatrics. 2013;132: S110-7.

16. Ontario Ministry of Community and Social Services. Accessibility for Ontarians with Disabilities Act [Internet]. Toronto (ON): Ontario Ministry of Community and Social Services; 2014 [cited 2014 Nov 24]. Available from: http://www.ontario.ca/laws/statute/05all.

17. Nurse-Family Partnership. Nurse-Family Partnership model elements [Internet]. Denver (CO): Nurse-Family Partnership National Service Office; [cited 2014 Nov 24]. Available from: http://www.nursefamilypartnership. org/communities/model-elements.

18. Jack SM, Busser LD, Sheehan D, Gonzalez A, Zwygers EJ, MacMillan HL. Adaptation and implementation of the Nurse-Family Partnership in Canada. Can J Public Health. 2012;103(Suppl 1):S42-8.

19. Ayala GX, Elder JP. Qualitative methods to ensure acceptability of behavioral and social interventions to the target population. J Public Health Dent. 2011;71(01):S69-S79.

20. Landy CK, Jack SM, Wahoush O, Sheehan D, MacMillan HL. NFP Hamilton Research Team. Mothers' experiences in the NurseFamily Partnership program: a qualitative case study. BMC Nurs. 2012;11:15.

21. British Columbia Ministry of Health and Ministry of Children and Family Development. Healthy minds, healthy people: a ten-year plan to address mental health and substance use in British Columbia. Victoria (BC): Government of British Columbia; 2010. 
22. Promote protect. prevent: our health begins here. B.C.'s guiding framework for public health [Internet]. Victoria (BC): Ministry of Health. 2013 [cited 2014 Nov 24]. Available from: http://www2.gov.bc.ca/gov/content/ health/about-bc-s-health-care-system/healthpriorities/bc-s-guiding-framework-for-publichealth.

23. Haynes L, Service O, Goldacre G, Torgerson D. Test, learn, adapt: developing public policy with randomized controlled trials. London (UK): Cabinet Office Behavioural Insights Team; 2012.

24. Craig P, Dieppe P, Macintyre S, Michie S, Nazareth I, Petticrew M. Developing and evaluating complex interventions: new guidance [Internet]. London (UK): Medical Research Council; 2008 [cited 2014 Nov 24]. Available from: http://www.mrc.ac.uk/documents/pdf/complex-interventions-guidance/

25. Creswell JW, Plano Clark VL. Designing and conducting mixed methods research. $2^{\text {nd }}$ ed. Thousand Oaks (CA): Sage Publications; 2011.

26. Felitti VJ, Anda RF, Nordenberg D, et al. Relationship of childhood abuse and household dysfunction to many of the leading causes of death in adults. The Adverse Childhood Experiences (ACE) Study.Am J Prev Med.1998;14(4):245-58.

27. Shonkoff JP, Garner AS. The Committee on Psychosocial Aspects of Child and Family Health. The lifelong effects of early childhood adversity and toxic stress. Pediatrics. 2012;129:e232-46.

28. Gunnar MR, Donzella B. Social regulation of the cortisol levels in early human development. Psychoneuroendocrinology. 2002; 27:199-220.

29. Repetti RL, Taylor SE, Seeman TE. Risky families: family social environments and the mental and physical health of offspring. Psychol Bull. 2002;128(2):330-66.

30. Danese A, McEwen BS. Adverse childhood experiences, allostatic, allostatic load, and age-related disease. Physiol Behav. 2012; 106(1):29-39.

31. Taylor SE, Karlamangla AS, Friedman EM, Seeman TE. Early environment affects neuroendocrine regulation in adulthood. Soc Cogn Affect Neurosci. 2011;6(2):244-51.
32. Shonkoff JP. Leveraging the biology of adversity to address the roots of disparities in health and development. Proc Natl Acad Sci U S A. 2012;109(2):17302-7. 